http://jmscr.igmpublication.org/home/

ISSN (e)-2347-176x ISSN (p) 2455-0450

crossref DOI: https://dx.doi.org/10.18535/jmscr/v8i6.71

Journal Of Medical Science And Clinical Research

\title{
Study the effectiveness of EMLA in alleviation of pain during BCG vaccination given at birth
}

\author{
Authors \\ Dr Hitender Gautam ${ }^{1}$, Dr Pancham Kuma ${ }^{2}$, Dr Priyanka ${ }^{3 *}$ \\ ${ }^{1}$ Paediatrician at Civil Hospital Rajgarh, CMO Sirmour, Himachal Pradesh, India \\ ${ }^{2}$ Assistant Professor Department of Paediatrics, IGMC, Shimla, Himachal Pradesh, India \\ ${ }^{3}$ Resident, Department of Community Medicine, IGMC, Shimla, Himachal Pradesh, India \\ *Corresponding Author \\ Dr Priyanka
}

\begin{abstract}
Background and Aims: Neonates routinely undergo painful cutaneous procedures as part of their medical treatment, and during vaccination. Effective pain management is a desirable standard of care for newborns and may potentially improve their clinical and neurodevelopmental outcomes. Neonatal pain should be assessed routinely using context-specific, validated and objective pain methods, despite the limitations of currently available tools.BCG vaccines are given to all newborns under universal immunization program and a major cause of pain in neonates. Lidocaine-prilocaine 5\% cream (EMLA) is a topical anaesthetic that may be useful for diminishing the pain from these procedures. So the present study was planned to know the effectiveness of EMLA in alleviating pain during BCG vaccination given at Birth.

Methods: A total of 280 neonates meeting inclusion criteria and received BCG vaccine at the time of birth were enrolled in the study. They were divided in two groups each with 140 participants. Group 1 had received BCG vaccine after application of EMLA Ihour prior to vaccine (case group) and group 2 received vaccine after application of placebo (control group). Pain was assessed on NIPS scale in both the groups immediately after immunization, at 30 second and at 60 seconds after immunization.

Results: NIPS scores at all the intervals were significantly lower when the vaccine was given after EMLA (Group 1) compare to group 2 who was immunized after placebo( $P$ value of $<0.0001)$

Conclusion: Pain perceived by the newborn after EMLA application during BCG vaccine was less as compared to the placebo group.

Keywords: Neonatal Infant Pain Scale Score (NIPS), BCG Vaccine, Intramuscular, EMLA (eutectic mixture of lidocaine and prilocaine).
\end{abstract}

\section{Introduction}

Pain is defined as an unpleasant sensory and emotional experience associated with actual or potential tissue damage. Pain in children is mostly underestimated all around the world and often sub optimally managed. ${ }^{1,2}$ Children experiences pain as a result of injury, illness, during medical procedures and vaccination.

Vaccination are considered as the safest and most effective way to prevent serious illness and death ${ }^{3}$. Vaccination prevent approximately 2.5 million death every year, globally ${ }^{4}$. These vaccines are the most common source of unavoidable repeated 
iatrogenic pain in children. Although children are repeatedly exposed to immunization pain, the need for immunization pain management is often under-recognized in the Maternal and Child Health Centre (MCHC)

Health care providers are not sensitize towards the pain in neonates. They consider, it's a benign procedure which require no intervention, on the contrary pain when left unmanaged, could have undesirable effects on children. These repeated injection may cause short-term and long-term effects. They also include physiological changes such as apnoea, bradycardia, tachycardia, skin colour changes, sweating palms, increased respiration rate and muscular tonicity, increased intracranial pressure and behavioural changes, crying, sleep disturbances, feeding problems. This lead tointense anxiety regarding vaccination that may result in non-adherence to the recommended vaccination schedule ${ }^{5,6}$.

Different techniques are evolved in last few decades to reduce pain during various interventions in neonates which includes nonpharmacological methods like distractions, (video, music, tactile, blowing) sucrose solution or breast milk, patient positioning, vaccination $\mathrm{pH}$ and pharmacological like lignocaine gel or spray, EMLA, opioids.

EMLA cream was in use from 1980 for relieving pain neonates and children. EMLA Cream (lidocaine $2.5 \%$ and prilocaine $2.5 \%$ ) is an emulsion in which the oil phase is a eutectic mixture of lidocaine and prilocaine in a ratio of $1: 1$ by weight. It is used to relieve pain during lumber puncture, intra venous, intra arterial cannulation, intra muscular injection. It was also used for reduction of pain during vaccination in infants and children. There are very few studies in which effect of EMLA was studied on newborn during vaccination. So the present study was conducted to know the effect of EMLA in reducing pain in newborn during vaccination.

\section{Aims and Objectives}

To study the effectiveness of EMLA in alleviating pain during BCG vaccination given at birth.

\section{Material and Methods}

The study was conducted in the department of paediatrics Kamla Nehru State Hospital for Mother and child Shimla.

This was a prospective and interventional randamized comparative double blinded study conducted among healthy full term newborns. The study was conducted over a period of from $1^{\text {st }}$ September, 2017 through $31^{\text {st }}$ December 2017.Study participants was randomly divided into two groups by using computer generated random numbers.

\section{Inclusion Criteria}

After taking consent from the mother healthy term newborn babies born and receive BCG vaccination at Kamla Nehru Hospital which a tertiary care hospital were enrolled in the present study.

\section{Exclusion Criteria}

1. Gestation less than 37 weeks, IUGR baby

2. New born with any kind of illness.

3. New born requiring any kind of supportive treatment after birth.

4. New born with any major congenital malformations.

5. New born to those mothers who receive any drug which cause CNS depression in the baby.

The socio demographic profile of the all the cases was recorded as per a structured case recording format. The newborns were randomly divided into two groups by using computer generated random numbers.

Group 1: Included newborn whom EMLA patch was applied one hour prior to hepatitis B vaccination and act as cases for the study.

Group 2: Included newborn whom placebo patch was applied one hour prior to hepatitis vaccination and considered as controls for group 1 .

Placebo patch used was identical to EMLA patch in its consistency, colour and odour. 
The parents, as well as observer was not aware of which treatment was received by the neonate making the study double blinded. The person involve in randomization was not involve further in the study.

Newborn was laid down on the radiant warmer during whole procedure and was breastfed half an before vaccination. Vaccine was given to those newborns that were at the 3-4 brazilton stage of arousal $^{7}$ on the same surrounding and at the same examination table. EMLA patch or placebo was applied before vaccination depending on the group assigned after randomization. All the injections will be administered by the same staff nurse gently. EMLA cream 1gm were uniformly applied to an area of 1 square inch at the vaccination area (left deltoid region) and covered with an occlusive dressing (Tegaderm) for 60 to 90 minute ,the dressing were removed before procedure, the skin were wiped dry. $0.1 \mathrm{ml}$ of BCG vaccine was be administered intradermaly at the left deltoid using a tuberculin syringe with $0.45 \mathrm{x} 13 \mathrm{~mm}$ needle.

The primary outcome measure neonatal pain had been assessed using Neonatal Infant Pain Scale
(NIPS), a reliable tool to assess neonatal pain ${ }^{[13]}$. NIPS score of zero was ensured before vaccination and used as baseline for further comparison. NIPS score was observed immediately after the vaccine, at 30 second, and at one minute after administration of vaccine.

\section{Statistical Analysis}

The data was entered, cleaned in MS EXCEL spreadsheet and analysis was done using epi info version 7.2.2

All the categorical variables were presented in frequency and percentage (\%) and continuous variables was presented as mean $\pm \mathrm{SD}$ and median. Quantitative variables was compared using Unpaired t-test/Mann-Whitney Test (when the data sets were not normally distributed.) between the two groups. Qualitative variables was compared using Chi-Square test /Fisher's exact test. A $p$ value of $<0.05$ was considered statistically significant.

\section{Results}

We enrolled a total of 280 participants in our study divided in two groups. Each group had 140 newborns.

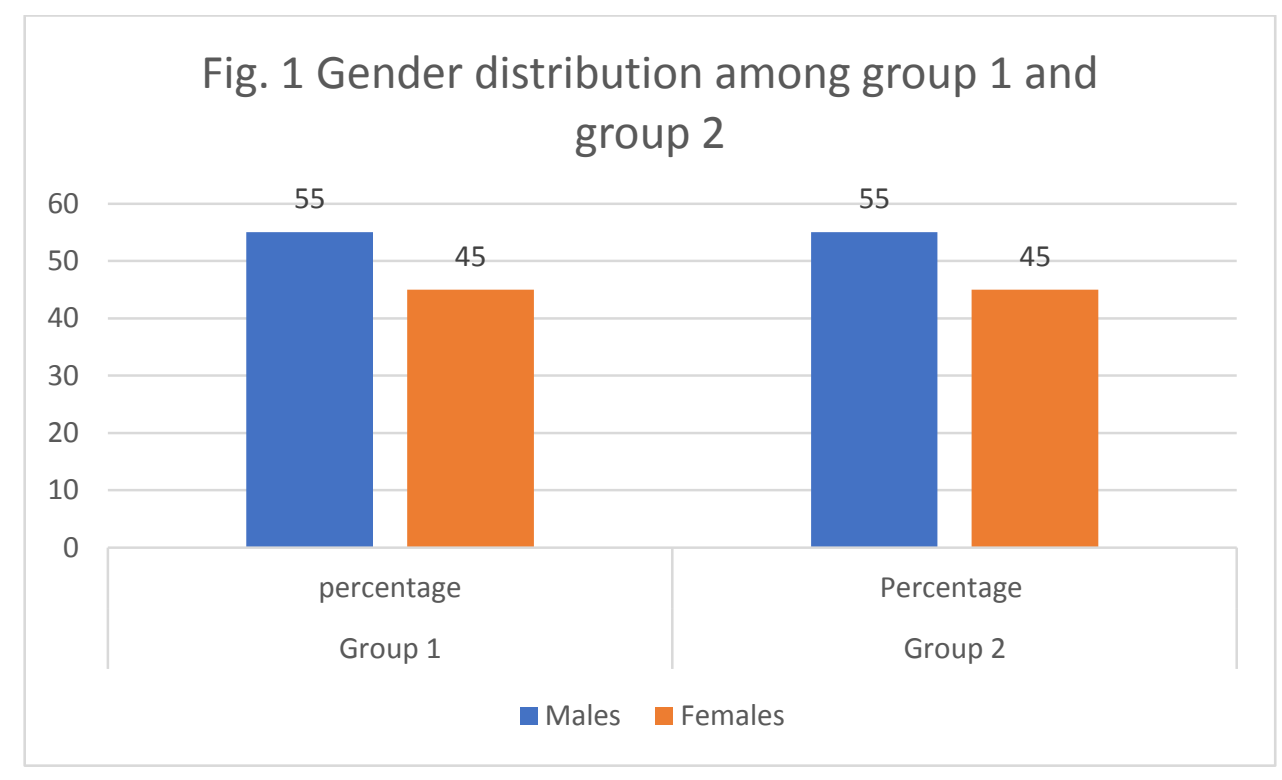

Fig. 1 in group 1.140 neonates were participated, out of which $55.00 \%$ were males and $45 \%$ were female as compared to group 2 in which out of 140 neonates $55 \%$ were male and $45 \%$ female. The difference of gender between two groups statistically not significant. Hence both the groups were comparable 
Table 2: Comparison of different variables present at birth between group 1 and group 2 given below:

\begin{tabular}{|l|c|c|c|c|}
\hline S.No & Variable & $\begin{array}{c}\text { Group 1 } \\
\text { Mean } \pm \text { SD }\end{array}$ & $\begin{array}{c}\text { Group 2 } \\
\text { Mean } \pm \text { SD }\end{array}$ & P value \\
\hline $\mathbf{1}$ & Birth weight & $2.80 \pm 0.29$ & $2.83 \pm 0.30$ & $\mathbf{0 . 3 0}$ \\
\hline $\mathbf{2}$ & Age in hours & $16.07 \pm 9.89$ & $16.64 \pm 9.63$ & $\mathbf{0 . 6 2}$ \\
\hline $\mathbf{3}$ & Gestational age & $38.37 \pm \mathbf{0 . 9 9}$ & $38.86 \pm 1.08$ & $\mathbf{0 . 3 1}$ \\
\hline $\mathbf{4}$ & OFC & $33.71 \pm 0.76$ & $33.39 \pm 0.65$ & $\mathbf{0 . 0 5}$ \\
\hline $\mathbf{5}$ & Length & $48.68 \pm 1.11$ & $48.05 \pm 1.06$ & $\mathbf{0 . 5 9}$ \\
\hline
\end{tabular}

Both the groups were comparable on the basis of demographic and anthropometric profile as mentioned in table 2 .

Table 3: Comparison of means of NIPS score of group 1 with group 2

\begin{tabular}{|l|c|c|c|}
\hline Group & $\begin{array}{c}\text { NIPS score at 0 seconds } \\
\text { Mean } \pm \text { SD }\end{array}$ & $\begin{array}{c}\text { NIPS score at 30 seconds } \\
\text { Mean } \pm \text { SD }\end{array}$ & $\begin{array}{c}\text { NIPS score at 60 seconds } \\
\text { Mean } \pm \text { SD }\end{array}$ \\
\hline BCG with EMLA(1) & $2.42 \pm 1.16$ & $0.71 \pm 0.83$ & $0.02 \pm 0.14$ \\
\hline BCG with Placebo $(2)$ & $3.32 \pm 1.73$ & $1.6643 \pm 1.66$ & $0.39 \pm 0.67$ \\
\hline P value & $<0.001$ & $<0.001$ & $<0.001$ \\
\hline
\end{tabular}

NIPS score was observed in group 1 and group 2 at $0 \mathrm{sec} 30$ seconds and 60 seconds after BCG vaccination. It was found that mean NIPS score in group 1(BCG vaccination after EMLA) was $2.42 \pm 1.15,0.71 \pm 0.83$ and $0.02 \pm 0.14$ which was significantly less as compared to group 2(BCG vaccine after placebo) where we observed the mean NIPS score was $3.32 \pm 1.73,1.66 \pm 1.66$ and $0.39 \pm 0.67$ at $0 \sec 30$ seconds and 60 seconds respectively with $\mathrm{p}$ value $<0.001$.

\section{Discussion}

Mean \pm SD of NIPS score in group 1(after application of EMLA before giving BCG vaccination ) at 0,30 and 60 seconds were $2.42 \pm 1.15,0.71 \pm 0.83$ and $0.02 \pm 0.14$ respectively and the mean of NIPS score of group 2 at 0,30 and 60 seconds were $3.32 \pm 1.73,1.66 \pm 1.66$ and $0.39 \pm 0.67$ respectively, which was significantly lower at all interval with $\mathrm{p}$ value of $<0.001$.It concluded that application of EMLA significantly decrease pain during BCG vaccine (intradermal injections). There is paucity of the studies about the effect OF EMLA in alleviating pain during the BCG vaccination. Most of the studies have been done to compare the effect of EMLA on intramuscular injections. So we are not able to compare our results with pain alleviation during interadermal vaccination. However, study done by
Taddio et al who used first cry and duration of cry as pain assessment tool reported a significant decrease in pain in the form of increase in the latency to first cry (MD, $0.90 \mathrm{sec} ; 95 \% \mathrm{CL} .46$ to 1.34, $\mathrm{P}<0.001)$ and decrease in duration of first cry $(\mathrm{MD},-1.30 \mathrm{sec} ; 95 \% \mathrm{Cl},-2.55$ to -0.05 ; $\mathrm{P}=0.04)$ for infant who received lidocain prilocaine versus a placebo during DPT vaccination at $6,10,14$ weeks, however they did not include newborns in their study.

Similarly, Uhari nurses also reported a lower mean VAS pain score (range, $0-10 \mathrm{~cm})(2.5 \mathrm{vs}$ 3.8; $\mathrm{P}<0.003$ ) and VAS crying score ( range, 0$10 \mathrm{~cm})(2.8 \mathrm{P}<0.003)$ for infant who received lidocaine-prilocaine then for those who received placebo during DPT vaccination in infant. They also did not include newborns in their study and used VAS score for assessment of pain.

In a study of lidocaine - prilocaine and no intervention, Dlli et al, reported a significant reduction in the NIPS score (range, 3-7) (MD, $4.0095 \% \mathrm{cCl},-4.83$ to -3.17 ; $\mathrm{P}<0.001)$ in infants 6 to 12 months of age and in the CHEOPS score (range , 4-13) (P .001; data =NR) during different vaccines Hep B at 0-2weeks, at 1 and 6 month, MMR at 9 months. 


\section{Conclusion}

Pain perceived during BCG vaccine was less when vaccination was done after EMLA application, which concluded that EMLA is effective modality in alleviating pain during vaccination through intradermal route.

\section{Recommendation}

EMLA is an effective pain alleviating modality and can be considered as pain reliving modalities during routine immunization. This will decrease immediate as well as late Neurodevelopmental complication in children due to repeated unavoidable pain during vaccination. This will also decrease parents anxiety regarding pain during vaccination and increase adherence to vaccination.

\section{References}

1. Paediatrics \& Child Health Division, The Royal Australasian College of Physicians. Guideline statement: management of procedure-related pain in children and adolescents. J Paediatr Child Health 2006; 42(suppl. 1): S1-S29

2. Taddio A, Manley J, Potash L, et al. Routine immunization practices: use of topical anesthetics and oral analgesics. Pediatrics 2007; 120: e637-e643.

3. Institute of Medicine (homepage on the internet). Childhood immunization schedule and safety : stakeholder concerns, scientific evidence, and future studies. Updated January 16,2013. Available from: http://www.iom.edu/reports/2013/The childhood -immunization-schedulesafety.aspx. Access July 14, 2014.

4. Brady K, Avner JR, Khine H. Perception and attitude of providers towards pain and anxiety associated with pediatric vaccine injection. Chn pediatr; 2011;50(2):140143.

5. Luthy KE, Beckstrand RL, Asay W, Hewett C. Vaccination parent experience vaccine anxiety too. J AM Assoc Nurse Pract. 2013;25(12):667-673.

6. Brazelton TB, Nugent JK. The Neurobehavioural Assessment Scale. 3 rd ed. London: MacKeith Press; 1995. 